\title{
ATIVIDADE FÍSICA NOS ESTUDANTES DE ENFERMAGEM: DETERMINANTES SOCIODEMOGRÁFICOS, ACADÉMICOS E DE PERSONALIDADE
}

\author{
PHYSICAL ACTIVITY IN NURSING STUDENTS: SOCIO-DEMOGRAPHIC, \\ ACADEMIC AND PERSONALITY DETERMINANTS \\ ATIVIDAD FÍSICA EN LOS ESTUDIANTES DE ENFERMERÍA: \\ DETERMINANTES SOCIODEMOGRÁFICOS, ACADÉMICOS Y DE \\ PERSONALIDAD
}

\author{
Carla Nunes (carlacgnunes@hotmail.com)* \\ Cláudia Chaves (claudiachaves21@gmail.com)** \\ João Duarte (jduarte@essv.jpv.pt)***
}

\section{RESUMO}

A inatividade física entre estudantes do ensino superior está associada a diversos fatores, tais como a complexidade e as exigências curriculares (Çiçek, 2018), especialmente em estudantes de enfermagem. Nesta perspetiva o objetivo desta investigação é identificar as variáveis sociodemográficas e académicas que interferem na prática de atividade física e determinar a associação entre a personalidade e a prática de atividade física nos estudantes de enfermagem. Realizou-se uma investigação quantitativa não experimental, em corte transversal, descritiva e correlacional, numa amostra não probabilística por conveniência de 372 estudantes de enfermagem. Aplicou-se um questionário de caracterização sociodemográfica, contexto académico, Questionário Internacional de Avaliação da Atividade Física (IPAQ) e o Inventário de Personalidade de Eysenck. Destacou-se que a amostra é maioritariamente feminina (86,3\%) e com idades até aos 20 anos $(41,7 \%)$. Os homens e os estudantes que frequentam os 2 o e 3 o anos registam maior prevalência de atividade física. As variáveis preditoras da atividade física foram a idade, o sexo e a personalidade; quanto mais velhos, menor a atividade física praticada pelos estudantes; há uma maior atividade física entre o sexo masculino e os de personalidade extrovertida. Aferiu-se que estudantes mais novos com personalidade extrovertida, praticam mais atividade física.

Palavras Chave: estudantes de enfermagem, atividade física, personalidade.

\section{ABSTRACT}

Physical inactivity amongst university students is related to several aspects, such as the complexity and curricula requirements (Çiçek, 2018), especially in nursing students. In this perspective the purpose of this investigation is to identify sociodemographic and academic variables that interfere in the practice of physical activity and determine the association between personality and the practice of physical activity in nursing students. A quantitative non-experimental, cross section, descriptive, 
and correlational research study was carried out, on a non-probabilistic sample, composed of 372 nursing students. A social-demographic characterization and academic context questionnaire was conducted, namely the International Physical Activity Questionnaires (IPAQ) and Eysenck's Personality Inventory. It was highlighted a predominantly feminine sample (86,3\%) aged 20 or below $(41,7 \%)$. Men as well as students who attend the second and third year have a predominance of physical activity. Predictor variables of physical activity were age, sex and personality; the older the students are, the lower physical activity they practice; there is a larger physical activity among men and the students with an outgoing personality. It was measured that younger students with an outgoing personality are those who practice more physical activity.

Keywords: nursing students, physical activity, personality.

\section{RESUMEN}

La inactividad física entre estudiantes de la enseñanza superior está asociada a diversos factores, así como la complejidad y las exigencias curriculares (Çiçek, 2018), especialmente en los estudiantes de enfermería. En esta perspectiva el objetivo de esta investigación es identificar las variables sociodemográficas y académicas que interfieren en la práctica de actividad física y determinar la asociación entre la personalidad y la práctica de actividad física en los estudiantes de enfermeira. Se realizó una investigación cuantitativa, no experimental, en sección transversal, descriptiva, y correlacional, en una muestra no probabilística por conveniencia de 372 estudiantes de enfermería. Se aplicó un cuestionario de caracterización sociodemográfica, contexto académico, Cuestionario Internacional de Evaluación de la Actividad Física (IPAQ) y el Inventario de Personalidad de Eysenck. Se destacó que la muestra es mayoritariamente femenina $(86,3 \%$ ) y con edades hasta los 20 años $(41,7 \%)$. Los hombres y los estudiantes de 2o y 3o años registran mayor prevalencia de actividad física. Las variables predictoras de la actividad física fueron la edad, el sexo y la personalidad; a maior edad, menor la actividad física practicada por los estudiantes; hay una mayor actividad física entre el sexo masculino y los de personalidad extrovertida. Se verificó que los estudiantes más jóvenes con personalidad extrovertida practican más actividad física.

Palabras-clave: estudiantes de enfermeira, actividad física, personalidad.

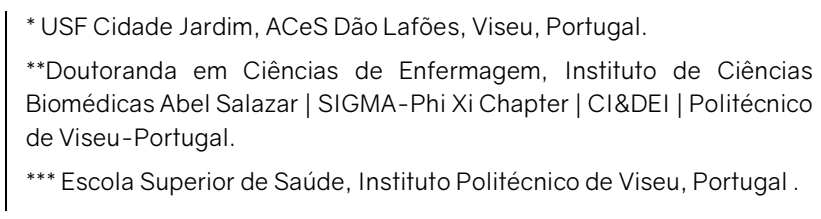

Submitted:22 $22^{\text {th }}$ July 2019

Accepted: $10^{\text {th }}$ October 2020 


\section{INTRODUÇÃO}

Os estudantes de enfermagem servem como modelos para promover comportamentos e estilos de vida saudáveis, no entanto, enfrentam múltiplos fatores indutores de stresse que podem levar à adoção de comportamentos de risco (Tavolacci, Delay, Grigioni, De'chelotte, \& Ladner, 2018). A entrada no ensino superior confronta o estudante com muitos desafios de caráter pessoal, desenvolvimental, social, académico e institucional que implicam alterações no seu padrão de comportamento, mais concretamente no que se refere à prática de atividade física, que assume um importante papel na vida das pessoas, quer no que respeita à aquisição e à manutenção da saúde, quer em relação à melhoria da condição física. Com o aumento da atividade física pode prevenir-se a obesidade e as doenças associadas e, ao nível psicológico, a redução dos níveis de depressão, melhoria da autoestima, controlo do stresse e da ansiedade, melhor interação com outras pessoas, fatores estes, que contribuem positivamente para uma melhor qualidade de vida dos estudantes de enfermagem (Richards, 2015; ValdesBadilla, Godoy-Cumillaf, Herrera Valenzuela \& Durán-Agüero, 2015; Sharry \& Timmins 2016; Primo, Molari, Luna, Bispo, Ossadad \& Costa, 2017; Nogueira, Ferreira, Rodrigues, Murano, Pereira \& Pereira, 2018). Para os mesmos autores, os estudantes de enfermagem apresentam baixos níveis de atividade física registando-se uma maior prevalência no sexo feminino, o que aumenta o risco para a sua saúde e pode influenciar as práticas de promoção de saúde. As pessoas fisicamente ativas são mais saudáveis e menos propensas a desenvolver doenças crónicas em comparação com as inativas, (Latino-Martel, Cottet, Druesne-Pecollo, Pierre, Touillaud, Touvier et al., 2016; Lopes, 2016; Hosseinzadeh, Heidari, Karbord, Azimian \& Alizadeh, 2017; Blake, Stanulewicz \& Mcgill, 2017).

A atividade física está diretamente associada à promoção de melhores padrões de vida e de saúde (Latino-Martel, Cottet, Druesne-Pecollo, Pierre, Touillaud, Touvier, et al., 2016). Face a esta realidade, este estudo abordou a atividade física em estudantes de enfermagem e os determinantes sociodemográficos e de contexto psicológico. Considerando o exposto, formularam-se as seguintes questões de investigação: Que variáveis sociodemográficas e académicas têm repercussão na prática de atividade física? Que associação existe entre a personalidade e a prática de atividade física? Decorrente das interrogações formuladas foram elaborados um conjunto de objetivos: Identificar as variáveis sociodemográficas e académicas que interferem na prática de atividade física; Determinar a associação entre a personalidade e a prática de atividade física.

\section{METODOLOGIA}

Neste estudo optou-se pelo tipo de investigação quantitativa não experimental, em corte transversal, descritiva e correlacional. Recorreu-se a uma amostragem não probabilística por conveniência que teve por base 372 estudantes de enfermagem de ambos os sexos distribuídos pelas diferentes escolas de enfermagem e saúde de Portugal Continental. Os estudantes apresentam uma idade mínima de 18 anos e uma máxima de 54 anos, $(M=23,33$ anos; $\pm 6,41$ anos).

A recolha de dados foi realizada através de um protocolo de avaliação online com parecer favorável da Comissão de Ética da Escola Superior de Saúde de Viseu (№ 1/2014) e abrangeu a Escola Superior de Saúde de Viseu e outras escolas superiores de enfermagem e saúde a 
nível nacional que se dispuseram a participar. Assegurou-se o anonimato e a confidencialidade dos dados.

O protocolo referido incluía um texto de apresentação com objetivos e esclarecimentos de preenchimento e o agradecimento pela colaboração prestada, seguindo-se um questionário de caracterização sociodemográfica e académica, e os seguintes instrumentos de avaliação: Questionário Internacional de Avaliação da Atividade Física (IPAQ) e Inventário de Personalidade de Eysenck (EPI).

O Questionário Internacional de Avaliação da Atividade Física - IPAQ - Versão Curta - foi desenvolvido por um grupo de investigadores elegidos pela Organização Mundial de Saúde (OMS), com características psicométricas aceitáveis para uso em estudos de prevalência sobre a participação em atividades físicas, sendo validado para a população portuguesa por Craig et al. (2003). Compreende três tipos de análise: (I) a avaliação da fiabilidade (testereteste) da mesma versão do IPAQ em dois momentos diferentes que mostrou um coeficiente de correlação de Spearman, $r_{s}=0,81$ (para um grau de significância de 95\%, IC 0,79-0,82) para a versão longa e $r_{s}=0,76$ também com grau de significância a 95\%, (IC 0,73-0,77) para a versão curta, sugerindo um bom grau de fiabilidade e bom nível de repetibilidade; (II) a validade de critério, comparando os dados auto-reportados aos avaliados por acelerómetro, durante 7 dias, apresentando um valor de correlação de $r=0,33(95 \%, I C$ 0,26-0,39) para a versão longa e $r=0,30(95 \%$, IC 0,23-0,36) para a versão curta, indicando uma concordância moderada entre as medidas subjetiva e objetiva de avaliação da atividade física; (III) validade concorrente (inter-método), pela comparação dos dados das versões longa e curta do IPAQ administradas no mesmo dia que sugere que as duas versões são semelhantes, ou seja, apresentam uma concordância razoável entre si, com uma correlação de Spearman de $r_{s}=0,67$ $(95 \%$; IC 0,64-0,70).

Apesar da diversidade das amostras e dos países envolvidos no referido estudo, os resultados sustentam a aceitabilidade das características psicométricas do IPAQ, testado quer em países desenvolvidos, quer em desenvolvimento, sobretudo em populações urbanas. Este instrumento contém 7 questões referentes à semana imediatamente anterior àquela em que os respondentes preenchem o questionário, mesmo que a pessoa não se considere fisicamente ativa. As questões incidem em atividades desenvolvidas na atividade profissional, académica e nas deslocações, atividades alusivas aos trabalhos domésticos e às atividades que a pessoa realizou no seu tempo livre para a recreação ou prática de exercício físico/desporto. Os respondentes devem considerar: atividades físicas vigorosas, as relativas às atividades que exigem um esforço físico intenso, resultando numa respiração ofegante; atividades físicas moderadas as que dizem respeito às atividades que requerem esforço físico moderado, resultando numa respiração um pouco mais forte do que o normal. Ao responderem às questões, os inquiridos apenas devem considerar as atividades físicas que realizaram durante pelo menos 10 minutos seguidos.

O Inventário de Personalidade de Eysenck é uma versão curta do Eysenck Personality Inventory que resultou de análises fatoriais e, à semelhança da sua versão original, mede as duas dimensões da personalidade: extroversão e neuroticismo. Está organizado em escala ordinal tipo Likert com 12 itens, cada um deles com quatro alternativas de resposta. Foi validado para a população portuguesa por Almiro e Simões (2013).

No que se refere ao tratamento estatístico recorreu-se a métodos da estatística descritiva e inferencial com recurso ao programa Statistical Package for the Social Sciences(SPSS) versão 21,0 (2013) para Windows. Quanto à estatística descritiva, como medidas estatísticas foram utilizadas frequências absolutas e percentuais, medidas de tendência central (média), 
medidas de dispersão (desvio padrão), e medidas de forma como assimetria (SK) e curtose (K). Foi igualmente utilizado o coeficiente de correlação de Pearson que é uma medida de associação linear usada para o estudo de variáveis quantitativas.

Para a análise inferencial usaram-se testes paramétricos e não paramétricos quando as condições para aplicação dos primeiros não se verificavam. Assim, foi utilizado o teste $\dagger$ de student ou teste de U-Mann-Whitney, para comparação de duas amostras, a análise de variância a um factor (ANOVA) ou Teste de Kruskal Wallis, para comparação de três ou mais amostras. O teste de Qui-quadrado $\left(\chi^{2}\right)$ foi utilizado para estudar relações entre variáveis nominais com duas ou mais categorias, comparando as frequências observadas com as que se esperavam obter no universo para se inferir sobre a relação existente entre as variáveis. Recorreu-se ainda à regressão linear múltipla, para avaliar o efeito das variáveis exógenas (independentes) como preditoras da variável endógena (dependente) (Marôco, 2014; Pestana \& Gageiro, 2014).

\section{RESULTADOS}

Resultante do somatório dos sete itens, que constituem o IPAQ, obteve-se um score global da prática da atividade física com um índice mínimo de 7 e um máximo de 21, sendo o score médio de $14,73( \pm 3,03)$. O coeficiente de variação $(20,57 \%)$ revela uma dispersão moderada em torno da média e os valores de assimetria e curtose, apresentavam curvas normocúrtica, todavia ligeiramente enviesada à direita.

Na tabela 1 é analisada a prevalência por item em função do sexo. Os dados inferenciais nela contidos, configuram a simulação de Monte Carlo para 10.000 amostras e com um nível de confiança "confidance level" a 95\%.

Em relação à primeira questão "nos últimos sete dias em quantos dias fez atividade física vigorosa" sobressaem os estudantes que praticaram atividade física moderada $(72,3 \%)$. Analisando os resultados por sexo afere-se que $73,2 \%$ das mulheres praticam atividade física moderada, enquanto que nos homens o percentual é de $66,7 \%$. Nos participantes que efetivamente realizaram atividade física intensa denota-se uma percentagem mais elevada para os homens $(21,6 \%$ vs $3,1 \%)$. Por outro lado nos que não realizaram atividade física vigorosa nos últimos sete dias, é notória a diferença entre homens e mulheres ( $23,7 \%$ vs $11,8 \%$ ). Os resíduos ajustados apontam para a existência de significância estatística para os homens que praticam atividade física intensa.

Quanto à média de tempo dedicado à prática de atividade física vigorosa, observa-se que mais de metade da amostra $(53,2 \%)$ dedica uma média de tempo elevada. Este percentual tende a aumentar quando comparamos ambos os sexos registando-se uma maior predominância no sexo masculino $(74,5 \%$ vs $49,8 \%)$. Ao invés, a média de tempo dedicado à atividade física classificada como fraca tende a ser mais elevada nas mulheres ( $45,5 \%$ vs $23,5 \%)$. Os resíduos ajustados indicam a existência de diferenças estatísticas significativas observada no grupo das mulheres com atividade física fraca e nos homens com atividade física elevada.

A segunda questão procurava saber "Quantos dias, nos últimos 7, fez atividades físicas moderadas" e nessa sequência a média de tempo dedicado à prática de atividades físicas moderadas. Quanto ao primeiro aspeto, 63,4\% da totalidade dos participantes praticam atividade física moderada a que corresponde cerca de 1-3 dias, por semana. Numa análise por sexo, este tipo de atividade é mais prevalente nas mulheres do que nos homens (64,5\% mulheres vs $56,9 \%$ homens). De registar que cerca de um terço da amostra não pratica 
atividade física moderada e 11,8\% faz atividade física moderada de forma intensa, isto é mais de três dias por semana. No que respeita à média de tempo dedicado à prática deste tipo de atividade prevalecem os estudantes tanto do sexo masculino como feminino que despendem um tempo superior a 49 minutos o que traduz uma média de tempo elevado $(54,2 \%$ mulheres vs 68,6\% homens). Os resíduos ajustados não apresentam significância estatística.

Analisando o "Tempo total gasto a caminhar nos dias em que andou, pelo menos 10 minutos seguidos nos últimos 7 dias", a maioria fê-lo entre $4-7$ dias (44,2\% mulheres vs $37,2 \%$ homens), traduzindo-se em atividade física elevada. Salienta-se ainda um percentual próximo entre ambos os sexos (32,4\% mulheres vs $35,4 \%$ homens), que neste tópico foram classificados como atividade moderada.

Quando questionados sobre o "tempo que passam sentados num dia normal" aferiu-se que estiveram entre 226-374 minutos ( 3 a 6 horas) sentados, num dia normal, correspondendo a atividade física moderada (39,9\% mulheres vs 43,1\% homens), conforme ilustra a tabela 1.

Tabela 1 - Prática de atividade física e sexo

\begin{tabular}{|c|c|c|c|c|c|c|c|c|}
\hline \multirow{2}{*}{$\begin{array}{l}\text { Sexo } \\
\text { Prática de atividade física }\end{array}$} & \multicolumn{2}{|c|}{ Feminino } & \multicolumn{2}{|c|}{ Masculino } & \multicolumn{2}{|c|}{ Total } & \multicolumn{2}{|c|}{ Residuais } \\
\hline & $\begin{array}{c}n= \\
(321)\end{array}$ & $\begin{array}{c}\% \\
(86,3)\end{array}$ & $\begin{array}{c}\mathrm{n} 0 \\
(51)\end{array}$ & $\begin{array}{c}\% \\
(13,7)\end{array}$ & $\begin{array}{c}\mathrm{n}= \\
(372)\end{array}$ & $\begin{array}{c}\% \\
(100,0)\end{array}$ & Fem. & Masc. \\
\hline \multicolumn{9}{|c|}{$\begin{array}{l}\text { Nos últimos } 7 \text { dias, em quantos dias fez atividade } \\
\text { física vigorosa }\end{array}$} \\
\hline Sem atividade & 76 & 23,7 & 6 & 11,8 & 82 & 22 & 1,9 & $-1,9$ \\
\hline Atividade moderada & 235 & 73,2 & 34 & 66,7 & 269 & 72,3 & 1,0 & $-1,0$ \\
\hline Atividade intensa & 10 & 3,1 & 11 & 21,6 & 21 & 5,6 & $-5,3$ & 5,3 \\
\hline \multicolumn{9}{|c|}{$\begin{array}{l}\text { Média de tempo dedicado à prática de atividades } \\
\text { físicas vigorosas }\end{array}$} \\
\hline Fraca & 146 & 45,5 & 12 & 23,5 & 158 & 42,5 & 2,9 & $-2,9$ \\
\hline Moderada & 15 & 4,7 & 1 & 2,0 & 16 & 4,3 & 0,9 & $-0,9$ \\
\hline Elevada & 160 & 49,8 & 38 & 74,5 & 198 & 53,2 & $-3,3$ & 3,3 \\
\hline \multicolumn{9}{|c|}{$\begin{array}{l}\text { Quantos dias, nos últimos 7, fez atividades físicas } \\
\text { moderadas }\end{array}$} \\
\hline Sem atividade & 79 & 24,6 & 13 & 25,5 & 92 & 24,7 & $-0,1$ & 0,1 \\
\hline Atividade moderada & 207 & 64,5 & 29 & 56,9 & 236 & 63,4 & 1,1 & $-1,1$ \\
\hline Atividade intensa & 35 & 10,9 & 9 & 17,6 & 44 & 11,8 & $-1,4$ & 1,4 \\
\hline \multicolumn{9}{|c|}{$\begin{array}{l}\text { Média de tempo dedicado à prática de atividades } \\
\text { físicas moderadas }\end{array}$} \\
\hline Fraca & 145 & 45,2 & 15 & 29,4 & 160 & 43,0 & 2,1 & $-2,1$ \\
\hline Moderada & 2 & 0,6 & 1 & 2,0 & 3 & 0,8 & $-1,0$ & 1,0 \\
\hline Elevada & 174 & 54,2 & 35 & 68,6 & 209 & 56,2 & $-1,9$ & 1,9 \\
\hline \multicolumn{9}{|c|}{$\begin{array}{l}\text { Tempo total gasto a caminhar nos dias em que } \\
\text { andou, pelo menos } 10 \text { minutos seguidos }\end{array}$} \\
\hline Fraca & 75 & 23,4 & 14 & 27,5 & 89 & 23,9 & $-0,6$ & 0,6 \\
\hline Moderada & 104 & 32,4 & 18 & 35,3 & 122 & 32,8 & $-0,4$ & 0,4 \\
\hline Elevada & 142 & 44,2 & 19 & 37,2 & 161 & 43,3 & 0,9 & $-0,9$ \\
\hline \multicolumn{9}{|c|}{ Tempo que passa sentado, num dia normal } \\
\hline Fraca & 107 & 33,3 & 11 & 21,6 & 118 & 31,7 & 1,7 & $-1,7$ \\
\hline Moderada & 128 & 39,9 & 22 & 43,1 & 150 & 40,3 & $-0,4$ & 0,4 \\
\hline Elevada & 86 & 26,8 & 18 & 35,3 & 104 & 28,0 & $-1,3$ & 1,3 \\
\hline
\end{tabular}

Tendo por base o score bruto da escala e os pontos de corte estabelecidos pelos autores foi possível classificar os participantes em três grupos a saber: inativos os que obtiveram pontuação inferior ou igual a 14 pontos, intermédios 15 pontos e ativos superior ou igual a 16 pontos. Os resultados foram analisados em função de algumas variáveis sociodemográficas de contexto académico e antropométricas. A tabela 2 reporta os dados verificando-se que a diferença entre a prevalência dos inativos $(42,7 \%)$ e a dos ativos $(43,8 \%)$ é de aproximadamente $10,0 \%$. 
Tabela 2 - Classificação dos inquiridos segundo a atividade física

\begin{tabular}{lcc}
\hline Classificação & no & \% \\
\hline Inativos & 159 & 42,7 \\
\hline Intermédios & 50 & 13,4 \\
\hline Ativos & 163 & 43,8 \\
\hline Total & $\mathbf{3 7 2}$ & $\mathbf{1 0 0 , 0}$ \\
\hline
\end{tabular}

Analisaram-se os resultados em função de algumas variáveis sociodemográficas de contexto académico e antropométricas.

Assim, relativamente ao sexo, verificou-se que os homens desenvolvem uma maior atividade física quando comparados com as suas congéneres sendo as diferenças estatisticamente significativas, conforme a tabela 3.

Tabela 3 - Relação entre a atividade física e sexo

\begin{tabular}{lccc}
\hline Sexo & OM & UMW & p \\
\cline { 1 - 2 } Feminino & 179,96 & 6086,000 & 0,003 \\
\hline Masculino & 227,67 & & \\
\hline
\end{tabular}

Analisada a prática da atividade física pelos estudantes em função da idade, anota-se que os estudantes com idade inferior ou igual a 20 anos são mais ativos ( $M=14,81 \pm 2,74)$, seguindose os que se situam na faixa etária entre os $21-22$ anos $(M=14,80 \pm 2,94)$, conforme a tabela 4 , mas as diferenças encontradas não são estatisticamente significativas.

Tabela 4 - Análise de variância da atividade física em função da idade

\begin{tabular}{lcccc}
\hline Idade & Média & dp & f & p \\
\hline$\leq 20$ anos & 14,81 & 2,74 & & \\
\cline { 1 - 2 } $21-22$ anos & 14,80 & 0,2219 & 0,804 \\
\cline { 1 - 2 }$\geq 23$ anos & 14,58 & 3,94 & & \\
\hline
\end{tabular}

O modo de deslocação de casa para a escola e vice-versa foi também objeto de análise. Compulsando os resultados obtidos com o teste † de Studente insertos na tabela 5, afere-se que os que se deslocam a pé obtiveram índices de atividade física mais elevado ( $M=15,08$ $\pm 2,81$ ), mas sem significância estatística.

Tabela 5 - Teste t para diferença de médias entre a atividade física e como se deslocam de casa para a escola

\begin{tabular}{lccccc}
\hline $\begin{array}{l}\text { Modo de deslocação de casa } \\
\text { para a escola }\end{array}$ & Média & dp & $\begin{array}{c}\text { Leven,s } \\
\mathbf{p}\end{array}$ & $\mathbf{f}$ & $\mathbf{p}$ \\
\hline A pé & 15,08 & 2,81 & 0,159 & 1,644 & 0,101 \\
\hline Veículo motorizado & 14,54 & 3,14 & 0,159 & \\
\hline
\end{tabular}

Uma outra variável em análise diz respeito ao índice de massa corporal. Verifica-se que os estudantes classificados como obesos $(O M=224,91)$, são ao invés do que seria expectável os que praticam mais atividade física, seguindo-se os classificados com baixo peso $(O M=208,83)$ sendo encontradas apenas significâncias marginais entre grupos, conforme tabela 6. 
Tabela 6 - Relação entre a atividade física e o IMC

\begin{tabular}{|c|c|c|c|}
\hline IMC & OM & $\chi^{2}$ & $p$ \\
\hline Baixo peso & 208,83 & \multirow{4}{*}{6,726} & \multirow{4}{*}{0,081} \\
\hline Eutrofia & 187,85 & & \\
\hline Pré-obesidade & 160,47 & & \\
\hline Obesidade & 224,91 & & \\
\hline
\end{tabular}

A tabela 7 reporta-se à relação entre ano de escolaridade que frequentam e prática de atividade física. Em termos de médias, os estudantes dos 2o e 3o anos são os que apresentam índices mais elevados de atividade física e os de menor atividade física correspondem aos que frequentam as pós-graduações/mestrados sendo que as diferenças encontradas entre os grupos são estatisticamente significativas.

Tabela 7 - Análise de variância da atividade física em função do ano em que está inscrito

\begin{tabular}{lllll}
\hline Ano em que está inscrito & Média & dp & f & p \\
\hline 1o ano & 14,20 & 2,98 & & \\
\cline { 1 - 2 } 2o ano & 15,24 & 2,54 & & \\
\hline 3o ano & 15,24 & 2,93 & \multirow{2}{*}{0,016} \\
\hline 4o ano & 14,85 & 3,06 & & \\
\hline Pós-graduação/mestrado & 13,78 & 3,52 & & \\
\hline
\end{tabular}

Submeteram-se as variáveis sociodemográficas idade e sexo (dummy) e a variável de contexto psicológica personalidade (introversão e extroversão) a uma regressão linear múltipla.

O outputgráfico do modelo ajustado indica que a idade, o sexo masculino e a componente da personalidade extroversão são preditoras da atividade física. Os coeficientes de regressão ou de trajetória foram para a idade $(\beta$ idade $=-0,18)$, para o sexo masculino ( $\beta$ sexo masculino $=0,17$ ) e para a extroversão ( $\beta$ extroversão=0,14). O modelo explica cerca de $9 \%$ da variação observada da atividade física nas diferentes variáveis.

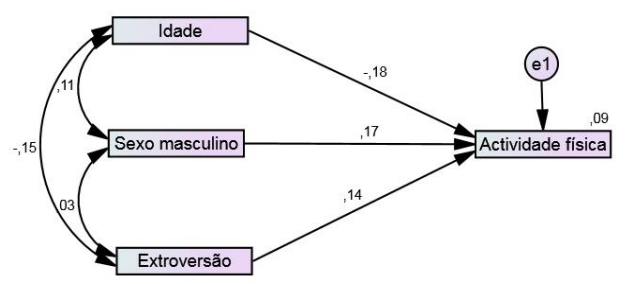

Figura 1 - Representação esquemática da regressão múltipla entre atividade física e variáveis sociodemográficas e da personalidade 


\section{DISCUSSÃO}

A amostra em estudo ( $n=372)$ é constituída maioritariamente por estudantes do sexo feminino na faixa etária $\leq 20$ anos. No que se refere ao Índice de Massa Corporal (IMC), evidenciam-se os estudantes de ambos os sexos com peso eutrófico. No global, os participantes deslocamse para a escola num veículo motorizado, demorando a maioria das mulheres $\leq 10$ minutos e dos homens $\geq 21$ minutos. No estudo de Blake, Stanulewicz e Mcgill (2016), que objetivou averiguar os níveis de atividade física de estudantes de enfermagem a maioria era feminina. De acordo com Fitzgerald (2015), vários estudos relacionados com a atividade física entre os estudantes de enfermagem sugerem que a maioria (78\%) não atende às orientações da atividade física recomendada, ou seja, 30 minutos ou mais de atividade física de intensidade moderada 5 dias por semana ou 20 minutos de atividade física de intensidade vigorosa 3 dias por semana para a promoção da sua saúde.

Na nossa amostra há evidências de estudantes de enfermagem ativos o que difere dos resultados apurados por Fitzgerald (2015) e Blake et al. (2016) que verificaram que a grande maioria dos estudantes de enfermagem, na sua amostra, eram inativos. Os autores Sharry e Timmins (2016) salientam que a transição para o ensino superior pode ter um efeito negativo na vida dos estudantes e nas escolhas do seu estilo de vida, resultando em ganho de peso, aumentando a inatividade e o stresse. Todavia, no presente estudo há uma prevalência de estudantes com peso eutrófico e ativos em termos de prática de atividade física, o que contraria os resultados dos referidos estudos.

Verificou-se que os estudantes do sexo masculino apresentam uma ordenação média superior em relação ao índice total da atividade física, o que resulta em diferença estatisticamente significativa $(p=0,003)$, corroborando os dados apurados por Lopes (2016). Klainin-Yobas, He e Lau (2015) salientam que, idealmente, os estudantes de enfermagem devem adotar comportamentos positivos de saúde dada a sua exposição a situações indutoras de stresse durante o seu percurso académico. No seu estudo, constaram que os estudantes do sexo masculino praticam mais atividade física do que os estudantes do sexo feminino.

Verificou-se que a variável idade não influenciou o índice de atividade física, todavia, são os estudantes com idade inferior ou igual a 20 anos que obtém o valor médio mais elevado, sendo estes os mais ativos, o que vai ao encontro dos dados apurados por Klainin-Yobas et al. (2015), pois também os seus resultados demonstram que os estudantes mais novos são os que praticam mais atividade física, sobretudo as vigorosas.

Outro resultado importante refere-se à variável ano em que os estudantes estão inscritos, com resultados estatisticamente significativos, sendo os estudantes dos 2 ㅇ e 3 o anos a revelarem maior índice de atividade física. No estudo de Lopes (2016), um terço dos estudantes revelaram um envolvimento e uma frequência da prática de atividade física inexistente, em ambos os sexos, e maioritariamente os que frequentavam o 1.o ano.

Confirma-se que, apesar da variável IMC não ter interferido estatisticamente no total do índice de atividade física, os estudantes com obesidade são os que referem praticar mais atividade física. Estes resultados podem justificar-se precisamente com o fator excesso de peso, ou seja, estes estudantes demonstram ter consciência dos benefícios da atividade física na redução do peso, o que os leva a tornar-se mais ativos fisicamente, optando por praticar mais atividade física. Os resultados do presente estudo confirmam os alcançados por Richards (2015), onde foram os estudantes de enfermagem com excesso de peso os que revelaram praticar mais atividade física. A prevalência de sobrepeso e da obesidade aumentou 
dramaticamente durante as últimas três décadas, com consequências negativas para a saúde pública. As estratégias recomendadas para reduzir a obesidade têm-se concentrado numa dieta mais saudável e prática de atividade física. A atividade física tem um potencial como parte da solução para a redução da obesidade (Wiklund, 2016).

A segunda questão de investigação presume verificar que associação existe entre a personalidade e a prática de atividade física, tendo-se verificado que a personalidade foi uma variável preditora da atividade física, ou seja, os estudantes mais extrovertidos praticam mais atividade física.

\section{CONCLUSÃO}

Concluiu-se do quadro concetual sobre a importância da prática de atividade física no desenvolvimento de estilos de vida saudáveis e no bem-estar geral dos indivíduos. Existe uma ampla evidência de que o exercício físico regular e moderado tem benefícios incontestáveis para a saúde física, psicológica e social, podendo contribuir significativamente para o bemestar geral da pessoa em qualquer faixa etária. Todavia, também se verificou que vários estudos concluíram que muitos estudantes do ensino superior, com a entrada na universidade, reduzem os níveis de atividade física, o que conduz a um estilo de vida cada vez mais sedentário.

Numa amostra de 372 estudantes de enfermagem, maioritariamente do género feminino, com idades inferiores ou iguais a 20 anos, o valor percentual mais elevado foi o dos estudantes que são ativos, com maior atividade física entre os homens, e os estudantes que frequentam os 2 은 e 3o anos. Concluiu-se também do estudo empírico que há maior atividade física por parte dos estudantes mais novos e com uma personalidade extrovertida.

As variáveis preditoras da atividade física foram a idade, o sexo e a personalidade; quanto mais velhos menor a atividade física praticada pelos estudantes; há uma maior atividade física entre o sexo masculino e os de personalidade extrovertida.

A prática da atividade física é benéfica à saúde e, por esse motivo, deve ser incentivada no ambiente universitário. Sabe-se que os estudantes estão continuamente sujeitos a trabalhos, prazos de entrega, longas horas de estudo, situações de exaustão física e mental, entre outras. Como tal, a implementação de programas de atividade física nos seus tempos livres poderá diminuir os desconfortos em relação à vida académica, com benefícios para a saúde física e psicológica. Além disso, estudar a atividade física de estudantes de enfermagem é importante para a descrição de aspetos relacionados com a saúde desse grupo, o que pode servir para a adoção de estratégias e programas de saúde na universidade, como medida preventiva para a adoção de hábitos de vida saudáveis e a melhoria da qualidade de vida, a curto e a longo prazo. A universidade deve servir para a disseminação de saberes, cultura e valores, dentre os quais, a atividade física não pode estar excluída.

\section{REFERÊNCIAS}

Almiro, P.A., \& Simões, M.R. (2013). Manual da versão portuguesa do Questionário de Personalidade de Eysenck - Forma Revista (EPQ-R). Coimbra: Laboratório de Avaliação Psicológica e Psicometria / Faculdade de Psicologia e de Ciências da Educação da Universidade de Coimbra 
Blake, H., Stanulewicz, N., \& Mcgill, F. (2016). Predictors of physical activity and barriers to exercise in nursing and medical students. JAN: Original Research: Empirical Research - Quantitative, 28, 917929. doi: 10.1111/jan.13181.Acedido em https://www.ncbi.nlm.nih.gov/pubmed/27731886

Blake, H., Stanulewicz, N., \& Mcgill, F. (2017). Predictors of physical activity and barriers to exercise in nursing and medical students. J Adv Nurs.; 73(4), 917-929. doi: 10.1111/jan.13181. Acedido em https://www.ncbi.nlm.nih.gov/pubmed/27731886

Çiçek, G. (2018). Quality of Life and Physical Activity among University Students. Universal Journal of Educational Research; 6(6), 1141-1148. DOI: 10.13189/ujer.2018.060602

Craig, C.L., Marshall, A.L., Sjöström, M., Bauman, A.E., Booth, M.L., Ainsworth, B.E., Pratt, M., Ekelund, U., Yngve, A., Sallis, J.F., \& Oja, P. (2003). International physical activity questionnaire: 12country reliability and validity. Med Sci Sports Exerc; 35: 1381-1395

FitzGerald, L.Z. (2015). Categorization and determinants of physical activity among nursing students. Journal of Nursing Education and Practice, Vol. 5, 5, 10-20. doi:https://doi.org/10.5430/jnep.v5n5p10, Acedido em http://www.sciedu.ca/journal/index.php/jnep/article/view/6127

Hosseinzadeh, K., Heidari, M.A., Karbord, A., Azimian, J., \& Alizadeh, A. (2017). Knowledge, Attitude and Practice Regarding Physical Activity in Nursing and Midwifery Students. Biotech Health Sci; 4(1), e35842, 2-5. doi: 10.17795/bhs-35842

Klainin-Yobas, P., He, H.G., \& Lau, Y. (2015). Physical fitness, health behaviour and health among nursing students: A descriptive correlational study. Nurse Education Today 35, 1199-1205. doi: 10.1016/j.nedt.2015.06.014. Acedido em https://www.ncbi.nlm.nih.gov/pubmed/26154132

Latino-Martel, P., Cottet, V., Druesne-Pecollo, N., Pierre, F., Touillaud, M., Touvier, M., Vasson, M.P., Deschasaux, M., Le Merdy, J., Barrandon, E., \& Ancellin, R. (2016). Alcoholic beverages, obesity, physical activity and other nutritional factors, and cancer risk: A review of the evidence. Critical Reviews in Oncology/Hematology:; 99, 308-323. https://doi.org/10.1016/j.critrevonc. 2016.01.002 PMID: 26811140

Lopes, F.M.F. (2016). Motivação e prática de exercício físico em estudantes do ensino superior. Um estudo realizado no Instituto Politécnico do Porto. (Tese de Doutoramento). Faculdade de Desporto da Universidade do Porto. Acedido em https://repositorioaberto.up.pt/bitstream/10216/112019/2/266644.pdf

Marôco, J. (2014). Análise estatística com o SPSS Statistics (6a ed.). Pêro Pinheiro: ReportNumbe

Nogueira, P.S., Ferreira, M.G., Rodrigues, P.R.M., Murano, A.P., Pereira, L.P., \& Pereira, R.A. (2018). Longitudinal Study on the Lifestyle and Health of University Students (ELESEU): design, methodological procedures, and preliminary results. Cadernos de Saúde Pública; 34(4): e00145917. http://www.scielo.br/pdf/csp/v34n4/1678-4464-csp-34-04-e00145917.pdf

Pestana, M. H., \& Gageiro, J. N. (2014). Análise de dados para as ciências sociais: a complementaridade do SPSS (6ạ ed.) Lisboa: Edições Silabo, Lda.

Primo, S.H., Molari, M., Luna, A.A., Bispo, N.N.C., Ossadad, V.A.Y., \& Costa, V.S.P. (2017). Avaliação dos níveis de atividade física em estudantes universitários do ensino a distância. J Health Sci; 19(2), 143-148. http://docs.bvsalud.org/biblioref/2017/12/876122/4907-17977-1-pb.pdf

Richards, E.A. (2015). The Evolution of Physical Activity Promotion Nurses can encourage patients to get more active, especially in small, incremental ways. 455614convertdoc.input.443066.mMooJ.doc

Sharry, P.Mc., \& Timmins, T. (2016). An evaluation of the effectiveness of a dedicated health and well being course on nursing students' health. Nurse Education Today, 44,26-32. doi: 10.1016/j.nedt.2016.05.004. Acedido em https://www.ncbi.nlm.nih.gov/pubmed/27429326 
Tavolacci, M.P., Delay, J., Grigioni, S., De'chelotte, P., \& Ladner, J. (2018). Changes and specificities in health behaviors among healthcare students over an 8-year period. PLOS ONE, 22, 1-18. Acedido em https://doi.org/10.1371/journal.pone.0194188 M

Valdes-Badilla, P., Godoy-Cumillaf, A., Herrera Valenzuela, T., \& Durán-Agüero, S. (2015). Comparación en hábitos alimentarios y condición física entre estudiantes de educación física y otras carreras universitárias. Nutr Hosp; 32, 829-836. http://dx.doi.org/10.3305/nh.2015.32.2.9194

Wiklund, P. (2016). The role of physical activity and exercise in obesity and weight management: Time for critical appraisal. Journal of Sport and Health Science, 5, 151-154. Acedido em https://ac.elscdn.com/S2095254616300060/1-s2.0-S2095254616300060-main.pdf?_tid=7b1a54c6-2f58$\underline{4270-87 f 5-7126 a d 9694 f e \& a c d n a t=1550605222 \_950 b 2 c b 1 a 1881 c e 7 d 32 e 24 f 809 d 72 c 3 e}$ 